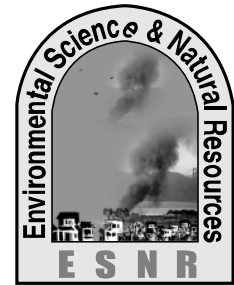

J. Environ. Sci. \& Natural Resources, 7(1): 121-125, 2014

ISSN 1999-7361

\title{
Development of Value Added Products From Home-Grown Lychee
}

\author{
S. Ahammed ${ }^{1}$, M. M. H. Talukdar ${ }^{1}$, M. S. Kamal ${ }^{2}$ \\ ${ }^{1}$ Department of Food Engineering and Technology \\ Hajee Mohammad Danesh Science and Technology University, Dinajpur-5200 \\ ${ }^{2}$ Department of Food Technology and Rural Industries \\ Bangladesh Agricultural University, Mymensingh-2202
}

\begin{abstract}
The experiment was concerned with developing of home-grown Lychee based different products, like squash and jelly to assess its prospect in respect to its marketability and food value. The fruits were collected from the local market and the pulp was extracted. Then the pulp was analyzed for proximate composition. The proximate analysis of lychee pulp showed $78.2 \%$ moisture, $17-18 \%$ TSS, $6.68 \%$ reducing sugar, $6.89 \%$ non-reducing sugar, $13.75 \%$ total sugar, $0.69 \%$ ash, $4.92 \mathrm{pH}, 0.327 \%$ acidity and $1.2 \mathrm{mg} / 100 \mathrm{gm}$ vitamin C. Products were stored at room temperatures $\left(28^{\circ} \mathrm{C}\right)$ and changes during storage were observed at an interval of 30 days for a period of 6 months. A testing panel consisting 10 panelists studied the acceptability of the samples. The consumer's preferences were measured by statistical analysis of the scores obtained from the response of the panel. Among the samples the following sample of squash, jelly was awarded the highest scores by the panelists; $\mathrm{S}-2$ respectively.
\end{abstract}

Key Words: Lychee squash, Lychee jelly, Preservation

\section{Introduction}

The lychee (Litchi chinensis) belongs to the family Sapindaceae and sub-family Nepheleae. It is one of the most important sub-tropical evergreen fruit trees which grow well in Bangladesh. Another member of the sub-family 'Anshphal' (Euphoria longana Lam.) also grows.

China is the leading county in terms volume production followed by India, Taiwan, Thailand and Viet name (FAO, 2002). Current production covers approximately 600000 ha, over $60 \%$ of which has been developed in the past 10 years. India is the second largest lychee producer, averaging approximately 500,000 tons of lychee annually on 56000ha. The third largest lychee producer is Taiwan (FAO, 2002).

A rich source of vitamin $\mathrm{C}$ litchi contains a fair amount of phosphorus, Calcium, Iron, Vitamin A and B. Litchi fruit contains $15.9 \%$ seed, $9.6 \%$ pericarp, $74.5 \%$ edible portion, $78.2 \%$ moisture, $1.2 \%$ acid, $0.97 \%$ oil, $0.94 \%$ protein, $0.69 \%$ ash, $6.89 \%$ free reducing sugars, $6.68 \%$ hydrolysable sugars and $13.75 \%$ total sugars (Ahmad, 1956).

Development of various value added products like jelly, juice and squash utilizing local producer is significantly important for expanding the country's ever growing food industries. In this study the prospect of using lychee fruit for the preparation of good quality squash and jelly with different composition along with their acceptability would be investigated.
On the basis of the above discussion, this study was under taken to achieve the following specific objectives:

- To prepare and analyzed squash and jelly.

- To assess the overall acceptability of the processed squash and jelly.

- To study shelf life in room temperature.

\section{Materials and Methods}

The indigenous lychee (Litchi chinesis) (used local variety name Bombai) and other raw materials were collected from the local market of Dinajpur. The major ingredients for the preparation of products were sugar, citric acid, pectin and KMS. These were used from the laboratory store. Brief descriptions of the methods followed for the study is given below:

\section{Extraction of lychee pulp}

The ripe and fresh lychee were washed thoroughly with potable water and then broken the upper layer of each lychee with maintaining hygienic condition before peeling. Water is added equal to the weight of the pulp and $\mathrm{pH}$ is adjusted by adding citric acid and heated at $80^{\circ} \mathrm{C}$ for one minute. The material is sieved through stainless steel sieve and large part of the fibers is removed. The pulp thus obtained is treated with KMS and preserved by freezing for future use.

\section{Formulation of Different Products}

The products prepared from lychee peels with different formulations were coded as; squash S-1, S-2, S-3 and Jelly E-1, E-2, E-3. The composition of different products was shown in the Table 1.1 and 1.2 respectively. 
Chemical Analysis: The processed samples were analyzed for their moisture, ash, acidity, vitamin $\mathrm{C}$, $\mathrm{pH}$, fat, protein and vitamin-C (AOAC, 2001)

Sensory evaluation: The consumer's acceptability of developed jelly and squash were evaluated by a taste-testing panel using hedonic rating test method. Samples were served to the panelists for characteristics color, flavor, texture and overall acceptability of processed Lychee Jelly and Lychee Squash.

The scale was arranged such that: $9=$ Like extremely, $8=$ like very much, $7=$ like moderately, $6=$ like slightly, $5=$ neither like nor dislike, $4=$ dislike slightly, $3=$ dislike moderately, $2=$ dislike very much and $1=$ dislike extremely.

Storage studies: Processed Lychee squash \& jelly was stored at ambient temperature $\left(27^{\circ} \mathrm{C}\right.$ to $\left.34^{\circ} \mathrm{C}\right)$ for a period of 6 months and quality parameters were assessed. During storage the changes in TSS, acidity, $\mathrm{P}^{\mathrm{H}}$, color, flavor, texture and vitamin $\mathrm{C}$ were observed. The analyses of the parameters were done according to standard analytical methods summarized by AOAC (1984) and Rangana (1994).

\section{Results and Discussion}

The proximate analysis of fresh lychee as moisture $78.20 \%$, TSS $17.00 \%$, Reducing sugar $6.68 \%$, Nonreducing sugar $6.89 \%$, ash $0.69 \%, \mathrm{p}^{\mathrm{H}} 4.92$, Acidity 0.327, Vitamin C $1.20 \mathrm{mg} / 100 \mathrm{gm}$.

Storage studies of Lychee products: Processed Lychee squash and jelly were stored at ambient temperature (27 to 34 ) ${ }^{\circ} \mathrm{C}$ for a period of 6 months and quality parameters were assessed as analytical methods summarized by AOAC (1984) and Rangana, (1994).

\section{Cost Analysis}

In this case the experiment was done with lychee of which the cost of raw material was analyzed for squash and jelly. The cost analysis of squash and jelly production from lychee is shown in Table 4.7 and Table 4.8 respectively

The panelists tasted the products and assigned marks for color, flavor, texture and overall acceptability within acceptable limit of ranking 'like slightly' to 'like very much'. The S-2 sample was more acceptable by the panelists and the E-2 sample is more acceptable.

Table 3: Storage studies of lychee squash

\begin{tabular}{|c|c|c|c|c|c|c|c|c|c|}
\hline \multirow{2}{*}{ 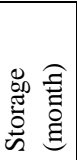 } & \multirow{2}{*}{ 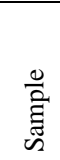 } & \multicolumn{3}{|l|}{ Observations } & \multirow{2}{*}{$\begin{array}{l}\text { TSS } \\
(\%)\end{array}$} & \multirow{2}{*}{$\begin{array}{l}\text { Acidity } \\
(\%)\end{array}$} & \multirow{2}{*}{$\mathrm{pH}$} & \multirow{2}{*}{$\begin{array}{l}\text { Vitamin-C } \\
(\mathrm{mg} / 100 \mathrm{gm})\end{array}$} & \multirow{2}{*}{ Remark } \\
\hline & & Color & Flavor & Turbidity & & & & & \\
\hline \multirow{3}{*}{0} & S-1 & Bright Whitish & Fresh & Clear & 40 & 1.25 & 3.00 & 1.25 & Good \\
\hline & S-2 & Bright Whitish & Fresh & Clear & 40 & 1.25 & 3.00 & 1.25 & Good \\
\hline & S-3 & Bright Whitish & Fresh & Clear & 40 & 1.25 & 3.00 & 1.25 & Good \\
\hline \multirow{3}{*}{1} & S-1 & Bright Whitish & Fresh & Clear & 40 & 1.25 & 3.00 & 1.25 & Good \\
\hline & S-2 & Bright Whitish & Fresh & Clear & 40 & 1.25 & 3.00 & 1.25 & Good \\
\hline & $S-3$ & Bright Whitish & Fresh & Clear & 40 & 1.25 & 3.00 & 1.25 & Good \\
\hline \multirow{3}{*}{2} & S-1 & Bright Whitish & Fresh & Clear & 40 & 1.30 & 2.90 & 1.20 & Good \\
\hline & S-2 & Bright Whitish & Fresh & Clear & 40 & 1.28 & 2.95 & 1.15 & Good \\
\hline & $\mathrm{S}-3$ & Pasty & Fresh & Clear & 40 & 1.28 & 2.95 & 1.15 & Fair \\
\hline \multirow{3}{*}{3} & S-1 & Bright Whitish & Fresh & Clear & 40 & 1.35 & 2.80 & 1.15 & Good \\
\hline & $\mathrm{S}-2$ & Bright Whitish & Fresh & Clear & 40 & 1.32 & 2.83 & 1.15 & Good \\
\hline & $S-3$ & Pasty & $\begin{array}{l}\text { Off } \\
\text { Flavor }\end{array}$ & Not Clear & 40 & 1.30 & 2.90 & 1.15 & Fair \\
\hline \multirow{3}{*}{4} & S-1 & Bright Whitish & Fresh & Clear & 40 & 1.42 & 2.64 & 1.05 & Good \\
\hline & S-2 & Bright Whitish & Fresh & Clear & 40 & 1.36 & 2.75 & 1.10 & Good \\
\hline & $S-3$ & Pasty & $\begin{array}{l}\text { Off } \\
\text { Flavor }\end{array}$ & Not Clear & 40 & 1.35 & 2.81 & 1.08 & Not Good \\
\hline \multirow{3}{*}{5} & S-1 & Bright Whitish & Fresh & Clear & 40 & 1.47 & 2.54 & 1.00 & Good \\
\hline & $\mathrm{S}-2$ & Bright Whitish & Fresh & Clear & 40 & 1.39 & 2.70 & 1.06 & Good \\
\hline & S-3 & Pasty & $\begin{array}{l}\text { Off } \\
\text { Flavor }\end{array}$ & Not Clear & 40 & 1.40 & 2.68 & 1.05 & $\begin{array}{l}\text { Not } \\
\text { Good }\end{array}$ \\
\hline \multirow{3}{*}{6} & S-1 & Pasty & $\begin{array}{l}\text { Off } \\
\text { Flavor }\end{array}$ & Not Clear & 40 & 1.53 & 2.41 & 1.00 & $\begin{array}{l}\text { Not } \\
\text { Good }\end{array}$ \\
\hline & $\mathrm{S}-2$ & Bright Whitish & Fresh & Clear & 40 & 1.42 & 2.65 & 1.02 & Fair \\
\hline & S-3 & Pasty & $\begin{array}{l}\text { Off } \\
\text { Flavor }\end{array}$ & Not Clear & 40 & 1.46 & 2.60 & 1.00 & Not Good \\
\hline
\end{tabular}


Table 4: Storage studies of lychee jelly

\begin{tabular}{|c|c|c|c|c|c|c|c|c|}
\hline \multirow{2}{*}{$\begin{array}{l}\text { Storage } \\
\text { (month) }\end{array}$} & \multirow{2}{*}{ Sample } & \multicolumn{3}{|c|}{ Observations } & \multirow{2}{*}{$\begin{array}{l}\text { TSS } \\
(\%)\end{array}$} & \multirow{2}{*}{$\begin{array}{l}\text { Acidity } \\
(\%)\end{array}$} & \multirow{2}{*}{$\mathrm{pH}$} & \multirow{2}{*}{ Remark } \\
\hline & & Color & Flavor & Turbidity & & & & \\
\hline \multirow{3}{*}{0} & E-1 & Watery & Fresh & Clear & 67.15 & 0.50 & 2.77 & Good \\
\hline & E-2 & Watery & Fresh & Clear & 67.15 & 0.50 & 2.77 & Good \\
\hline & E-3 & Watery & Fresh & Clear & 67.15 & 0.50 & 2.77 & Good \\
\hline \multirow{3}{*}{1} & E-1 & Watery & Fresh & Clear & 67.15 & 0.50 & 2.77 & Good \\
\hline & E-2 & Watery & Fresh & Clear & 67.15 & 0.50 & 2.77 & Good \\
\hline & E-3 & Watery & Fresh & Clear & 67.15 & 0.50 & 2.77 & Good \\
\hline \multirow{3}{*}{2} & E-1 & Watery & Fresh & Clear & 67.15 & 0.58 & 2.70 & Good \\
\hline & E-2 & Watery & Fresh & Clear & 67.15 & 0.58 & 2.70 & Good \\
\hline & E-3 & Watery & Fresh & Clear & 67.15 & 0.54 & 2.71 & Good \\
\hline \multirow{3}{*}{3} & E-1 & Watery & Fresh & Clear & 67.15 & 0.64 & 2.59 & Good \\
\hline & E-2 & Watery & Fresh & Clear & 67.15 & 0.62 & 2.60 & Good \\
\hline & E-3 & Watery & Fresh & Clear & 67.15 & 0.60 & 2.63 & Good \\
\hline \multirow{3}{*}{4} & E-1 & Blush & Fresh & Not Clear & 67.15 & 0.70 & 2.54 & Fair \\
\hline & E-2 & Watery & Fresh & Clear & 67.15 & 0.66 & 2.57 & Good \\
\hline & E-3 & Watery & Fresh & Clear & 67.15 & 0.60 & 2.64 & Good \\
\hline \multirow{3}{*}{5} & E-1 & Blush & Off Flavor & Not Clear & 67.15 & 0.72 & 2.51 & Not Good \\
\hline & E-2 & Watery & Fresh & Clear & 67.15 & 0.69 & 2.55 & Good \\
\hline & E-3 & Watery & Fresh & Clear & 67.15 & 0.66 & 2.58 & Fair \\
\hline \multirow{3}{*}{6} & E-1 & Blush & Spoiled & Not Clear & 67.15 & 0.80 & 2.41 & Spoiled \\
\hline & E-2 & Watery & Fresh & Clear & 67.15 & 0.70 & 2.53 & Good \\
\hline & E-3 & Blush & Off Flavor & Not Clear & 67.15 & 0.72 & 2.50 & Not Gooc \\
\hline
\end{tabular}

Table 5: Duncan's Multiple Range Test (DMRT) for color, flavor, texture and overall acceptability of lychee squash.

\begin{tabular}{|c|c|c|c|c|}
\hline \multirow[t]{2}{*}{ Sample Code } & \multicolumn{4}{|c|}{ Sensory Attributes } \\
\hline & Color & Flavor & Texture & $\begin{array}{c}\text { Overall } \\
\text { Acceptability }\end{array}$ \\
\hline S-1 & $7.80^{\mathrm{a}}$ & $6.40^{\mathrm{b}}$ & $7.1^{\mathrm{ab}}$ & $6.70^{\mathrm{ab}}$ \\
\hline S-2 & $7.20^{\mathrm{a}}$ & $7.40^{\mathrm{a}}$ & $7.30^{\mathrm{a}}$ & $7.30^{\mathrm{a}}$ \\
\hline S-3 & $7.10^{\mathrm{a}}$ & $7.10^{\mathrm{a}}$ & $6.20^{\mathrm{b}}$ & $6.20^{\mathrm{b}}$ \\
\hline $\mathrm{LSD}(\mathrm{P}<0.01)$ & 0.877 & 0.754 & 0.940 & 0.819 \\
\hline
\end{tabular}

Table 6: Duncan's Multiple Range Test (DMRT) for color, flavor, texture and overall acceptability of Lychee jelly

\begin{tabular}{|l|l|l|l|l|}
\hline \multirow{2}{*}{ Sample Code } & \multicolumn{4}{c|}{ Sensory Attributes } \\
\cline { 2 - 5 } & \multicolumn{1}{|c|}{ Color } & \multicolumn{1}{c|}{ Flavor } & \multicolumn{1}{c|}{ Texture } & \multicolumn{1}{c|}{ Overall Acceptability } \\
\hline E-1 & $7.20^{\mathrm{a}}$ & $6.90^{\mathrm{b}}$ & $6.60^{\mathrm{b}}$ & $6.10^{\mathrm{b}}$ \\
\hline E-2 & $7.10^{\mathrm{a}}$ & $7.70^{\mathrm{a}}$ & $7.70^{\mathrm{a}}$ & $7.60^{\mathrm{a}}$ \\
\hline E-3 & $7.30^{\mathrm{a}}$ & $6.70^{\mathrm{b}}$ & $6.20^{\mathrm{b}}$ & $6.50^{\mathrm{b}}$ \\
\hline LSD $(\mathrm{P}<0.01)$ & 1.053 & 0.736 & 1.058 & 0.975 \\
\hline
\end{tabular}

\section{Cost Analysis}

In this case the experiment was done with lychee of which the cost of raw material was analyzed for squash and jelly. The cost analysis of squash and jelly production from lychee is shown in Table 4.7 and Table 4.8 respectively 
Table 7: Cost of 1 bottle (250gm) lychee Squash with Fixed Cost \& Break-Even Point

\begin{tabular}{|c|c|c|c|}
\hline \multirow{2}{*}{$\begin{array}{l}\text { No. } \\
{[A]}\end{array}$} & \multirow{2}{*}{$\begin{array}{l}\text { Particulars } \\
\text { Sales or Market price of fruit squash }(250 \mathrm{gm})\end{array}$} & \multicolumn{2}{|c|}{ Amount in Tk. } \\
\hline & & & 75.00 \\
\hline \multirow[t]{8}{*}{ [B] } & Variable cost & & \multirow{8}{*}{48.00} \\
\hline & Lychee pulp/juice & 5.00 & \\
\hline & Sugar & 5.00 & \\
\hline & Citric acid/Vinegar & 1.00 & \\
\hline & KMS (preservative) & 1.00 & \\
\hline & Electricity & 18.00 & \\
\hline & Labor & 14.00 & \\
\hline & Packaging & 4.00 & \\
\hline$[\mathbf{C}]$ & Contribution $[\mathrm{A}]-[\mathrm{B}]$ & & 27.00 \\
\hline [D] & Total Fixed cost ( $40 \%$ of total cost) & & 19.00 \\
\hline \multirow[t]{2}{*}[\mathrm{E}]{} & Total Cost [B]+[D] & & 67.00 \\
\hline & Profit $[A]-[E]$ & & 8.00 \\
\hline$[\mathbf{P}]$ & Break-Even Point $[\mathrm{D}] /[\mathrm{C}]$ & & $70 \%$ \\
\hline
\end{tabular}

Table 8: Cost of 1 bottle (250gm) lychee jelly with fixed cost \& break-even point

\begin{tabular}{|c|c|c|c|}
\hline No & Particulars & \multicolumn{2}{|c|}{ Amount in Tk. } \\
\hline$[\mathbf{A}]$ & Sales or Market price of fruit jelly (250gm) & & 120.00 \\
\hline \multirow[t]{8}{*}{ [B] } & Variable costs & & \multirow{8}{*}{78.01} \\
\hline & Lychee pulp/juice & 32.00 & \\
\hline & Sugar & 8.76 & \\
\hline & Citric acid/Vinegar & 0.25 & \\
\hline & KMS (preservative) & 1.00 & \\
\hline & Electricity & 18.00 & \\
\hline & Labor & 14.00 & \\
\hline & Packaging & 4.00 & \\
\hline [C] & Contribution $[\mathrm{A}]-[\mathrm{B}]$ & & 41.99 \\
\hline [D] & Total Fixed cost ( $40 \%$ of total variable cost) & & 31.20 \\
\hline \multirow[t]{2}{*}{ [E] } & Total Cost $[\mathrm{B}]+[\mathrm{D}]$ & & 109.21 \\
\hline & Profit $[\mathrm{A}]-[\mathrm{E}]$ & & 11.00 \\
\hline$[\mathbf{P}]$ & Break-Even Point [D] / [C] & & $74.30 \%$ \\
\hline
\end{tabular}

Table 1.1 Ingredients used to manufacture 250 gm of lychee squash

\begin{tabular}{|c|c|c|c|c|c|}
\hline \multirow[t]{2}{*}{ Sample } & \multicolumn{5}{|c|}{ Ingredient } \\
\hline & $\begin{array}{l}\text { Juice/ Pulp } \\
\text { (gm) }\end{array}$ & $\begin{array}{l}\text { Sugar } \\
(\mathrm{gm})\end{array}$ & $\begin{array}{l}\text { Preservative (KMS) } \\
\text { (gm) }\end{array}$ & $\begin{array}{l}\text { Citric Acid } \\
\text { (gm) }\end{array}$ & $\begin{array}{l}\text { Water } \\
(\mathrm{gm})\end{array}$ \\
\hline S-1 & 62.5 & 86.32 & 0.15 & 2.9 & 98.13 \\
\hline S-2 & 62.5 & 86.38 & 0.10 & 2.9 & 98.13 \\
\hline S-3 & 62.5 & 87.1 & - & 2.9 & 98.13 \\
\hline
\end{tabular}

Table 1.2 Ingredients used to manufacture 250 gm of lychee Jelly

\begin{tabular}{|l|l|l|l|l|l|}
\hline \multirow{2}{*}{ Sample } & \multicolumn{2}{|c|}{ Ingredient } \\
\cline { 2 - 7 } & $\begin{array}{c}\text { Juice/Pulp } \\
(\mathrm{gm})\end{array}$ & $\begin{array}{c}\text { Sugar } \\
(\mathrm{gm})\end{array}$ & $\begin{array}{c}\text { Citric Acid } \\
(\mathrm{gm})\end{array}$ & $\begin{array}{c}\text { Pectin } \\
(\mathrm{gm})\end{array}$ & $\begin{array}{c}\text { KMS } \\
(\mathrm{gm})\end{array}$ \\
\hline E-1 & 395.75 & 151.25 & 1.25 & 3.75 & 0.15 \\
\hline E-2 & 395.75 & 151.25 & 1.25 & 2.50 & 0.15 \\
\hline E-3 & 395.75 & 151.25 & 1.25 & 1.25 & 0.15 \\
\hline
\end{tabular}


Table 2: Chemical composition of lychee products

\begin{tabular}{|l|c|c|}
\hline \multirow{2}{*}{ Compositions } & \multicolumn{2}{|c|}{ Product } \\
\cline { 2 - 3 } & Squash & Jelly \\
\hline Moisture (\%) & 40.00 & 28.23 \\
\hline Ash (\%) & 0.38 & 0.29 \\
\hline Acidity (\%) & 1.25 & 2.50 \\
\hline $\mathrm{P}^{\mathrm{H}}$ & 3.00 & 13.85 \\
\hline Reducing Sugar (\%) & 13.13 & 50.76 \\
\hline Non-reducing Sugar (\%) & 27.54 & 64.61 \\
\hline Total Sugar (\%) & 40.67 & 67.15 \\
\hline TSS (\%) & 40.00 & - \\
\hline Vitamin-C (mg/100g) & 1.25 & \\
\hline
\end{tabular}

\section{References}

Ahmed. M. 1988. Effect of different preservatives on the retention of natural and added ascorbic acid in Orange squash. Pakistan J. Agric. CFTRI, 1972. Home Scale Preservation of fruits and Vegetables, $6^{\text {th }}$ Edition, India. Res. 9(2)

AOAC, 1984. Official Methods of Analysis of the Association of Official Analytical Chemist. 18th Ed. Washington D.C.

AOAC, 2001. Official Methods of Analysis of the Association of Official Analytical Chemist. 18th Ed. Washington D.C.
FAO. 2002. Lychee production in the asia pacific region. Food and Agriculture Organization of the United Nation. Bangkok, Thailand.

Rahman, A. 1964. Factors affecting the stability of vitamin $\mathrm{C}$ in tropical fruit juices and nectars, J. Agri. Uni. of Pakistan.

Rangana, S. 1994. Hand book of analyses of quality control for fruit and vegetable product. $4^{\text {th }}$ Ed. Tata McGraw Hill Pub. Co. Ltd. New Delhi. India. 